\title{
Three-Dimensional Coherent Bragg Imaging of Rotating Nanoparticles
}

\author{
Alexander Björling $\odot^{*}$ \\ MAX IV Laboratory, Lund University, 22100 Lund, Sweden \\ Lucas A. B. Marçalø \\ Synchrotron Radiation Research and NanoLund, Lund University, 22100 Lund, Sweden \\ José Solla-Gullón (1) \\ Institute of Electrochemistry, University of Alicante, 03080 Alicante, Spain \\ Jesper Wallentin $\odot$ \\ Synchrotron Radiation Research and NanoLund, Lund University, 22100 Lund, Sweden \\ Dina Carbone $\odot$ \\ MAX IV Laboratory, Lund University, 22100 Lund, Sweden \\ Filipe R. N. C. Maia $\odot$ \\ Department of Cell and Molecular Biology, Uppsala University, 75124 Uppsala, Sweden
}

(Received 19 July 2020; accepted 4 November 2020; published 9 December 2020)

\begin{abstract}
Bragg coherent diffraction imaging is a powerful strain imaging tool, often limited by beam-induced sample instability for small particles and high power densities. Here, we devise and validate an adapted diffraction volume assembly algorithm, capable of recovering three-dimensional datasets from particles undergoing uncontrolled and unknown rotations. We apply the method to gold nanoparticles which rotate under the influence of a focused coherent $\mathrm{x}$-ray beam, retrieving their three-dimensional shapes and strain fields. The results show that the sample instability problem can be overcome, enabling the use of fourth generation synchrotron sources for Bragg coherent diffraction imaging to their full potential.
\end{abstract}

DOI: $10.1103 /$ PhysRevLett.125.246101

Introduction.-Bragg coherent diffraction imaging (BCDI) has emerged as a promising technique for threedimensional strain imaging of crystalline nanoparticles $[1,2]$. The method is based on the iterative phase retrieval of a set of two-dimensional diffraction patterns, which together form a volume of intensities [3]. Phased diffraction volumes provide detailed three-dimensional maps of how strain and defects are distributed within single particles $[4,5]$. While it offers a lower resolution than electron microscopy or scanning probe methods, BCDI exploits the penetration depth and high strain sensitivity of $\mathrm{x}$ rays and is therefore applicable to demanding environments such as catalysts in working reactors or cells [6-14]. As $\mathrm{x}$-ray sources develop worldwide and higher coherent flux densities are made available, smaller particles can in principle be studied. Unfortunately, particle stability under the intense beam often becomes a limiting factor in practice [15].

In BCDI experiments [cf Fig. 1(a)], three-dimensional shape and strain information is encoded in a small region of reciprocal space surrounding each reciprocal lattice point $\mathbf{G}_{h k l}$. Because the reciprocal lattice rotates with the particle, and since the vector $\mathbf{G}_{h k l}$ provides a long lever arm, the entire diffraction volume can be recorded by rotating the particle through a small angular range $\delta \theta$ (see Ref. [3] for a detailed account of the reciprocal geometry). These socalled rocking curves typically cover angles on the order of a degree [16]. Similarly, depending on the size of the detector used, small rotations $\delta \omega$ of the particle roll angle can quickly rotate the Bragg peak off the detector area, while comparable rotations of the angle $\varphi$ leave the diffraction patterns essentially unaltered. Because of this angular sensitivity, any uncontrolled rotations due to local heating or to radiation pressure [17,18] make controlled BCDI experiments of small particles very difficult. An approach to deal with small deviations from nominal rocking angles has been developed [19]. The method which reformulates the phase retrieval problem is limited to small deviations from an ideal reciprocal space sampling pattern, and therefore optimized to deal with slow drifts and instrumental errors. In contrast, we present an approach that provides a solution for large uncontrolled particle rotations, where no prior knowledge of the angular trajectories across the rocking curve exists.

We have observed entirely uncontrolled rotations of $60 \mathrm{~nm}$ truncated-octahedral gold nanoparticles under the 
(a)

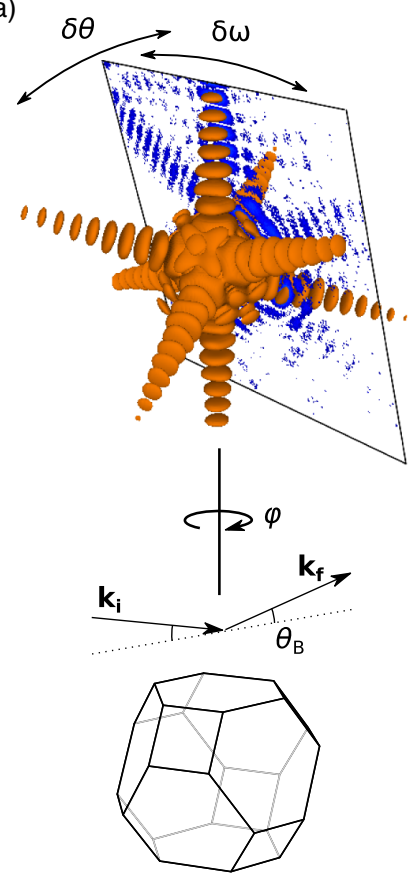

(b)

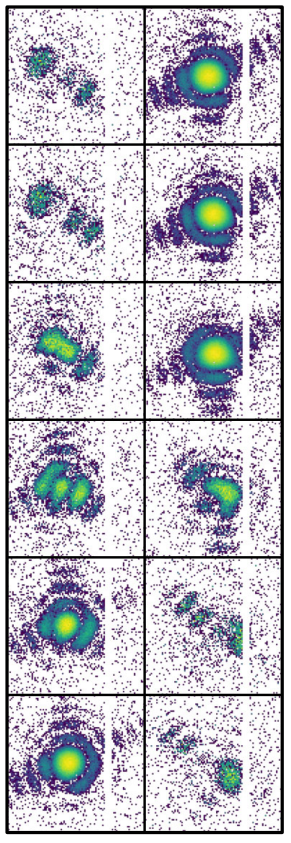

(c)

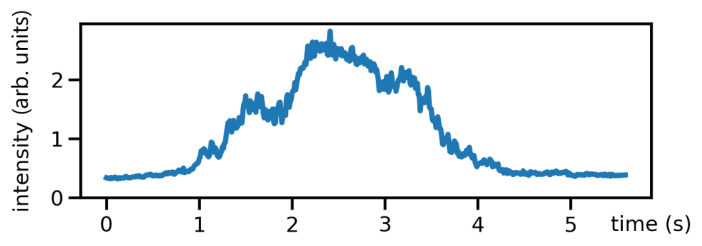

FIG. 1. (a) Geometrical description of the BCDI experiment. An example model particle (bottom) and its corresponding diffraction volume (top), which is sampled across the detector plane. Measured intensities are sensitive to small variations of the rocking $(\theta)$ and roll $(\omega)$ angles, but less sensitive to similar variations of the azimuth $(\varphi)$. (b) Diffraction frames recorded as a real particle rotates through the Bragg condition, with $48 \mathrm{~ms}$ exposure time. They correspond to cuts through the diffraction volume in (a) at unknown $\theta$ and $\omega$. The white vertical line is a gap between two detector modules. (c) The total diffracted intensity of a particle undergoing an uncontrolled rocking curve, recorded at $100 \mathrm{~Hz}$.

focused beam at the NanoMAX beam line of the MAX IV Laboratory, as exemplified in Fig. 1(b). The $10 \mathrm{keV}$ beam delivers around $2 \times 10^{10}$ coherent photons per second in a spot of around $100 \times 100 \mathrm{~nm}^{2}$ [20]. At this power density, these particles tend to rotate in and out of the diffraction condition on a timescale of seconds, completing inadvertent rocking curves around the selected reciprocal lattice point $\mathbf{G}_{111}$ in the process. As shown in Fig. 1(c), the intensity of the Bragg peak typically varies continuously but seemingly randomly between frames. Despite this, we here present a method capable of assembling data from unknown trajectories of $\theta$ and $\omega$ into coherent diffraction volumes.

The results obtained demonstrate that reliable threedimensional shape and strain images can be reconstructed

from these data, assuming that the diffraction volume is sufficiently oversampled along the rocking curve. By taking advantage of beam-induced particle instability and the ensuing rotations, turning them into a resource for a more efficient data acquisition without the need for rotation motors or energy scans, we thereby show that BCDI is possible without control or even knowledge of the rocking and roll angles. This represents a substantial advance in the field of single-particle inverse microscopy, opening new avenues for the exploitation of the increased coherence properties of x-ray sources.

Diffraction volume assembly.-The present problem of assembling slices from unknown positions in a diffraction volume has a counterpart in the forward three-dimensional diffraction imaging performed at $\mathrm{x}$-ray free-electron lasers (XFELs) [21,22]. In the forward geometry, all slices intersect the reciprocal space origin at three unknown angles. In the Bragg case, the slices do not intersect but can be considered virtually parallel, offset by differences in $\theta$ and $\omega$. Another key difference is that in the XFEL case, each diffraction pattern is collected from a different particle in a diffract-and-destroy manner [23], and assembly assumes that the original particles were identical. In the present case, a whole series of diffraction patterns such as that in Fig. 1(b) is collected from one single particle.

Inspired by the expand-maximize-compress algorithm used for the single-shot XFEL technique [24], we have developed an analogous algorithm for BCDI. In essence, a model $W$ for the diffraction intensity volume is iteratively updated based on likelihood maximization with respect to the frames and their positions in $\theta$ and $\omega$. At the same time, the diffraction volume is constrained to be compatible with a particle of limited extent. Optionally, continuity of the parameters $\theta(k)$ and $\omega(k)$ as functions of time can also be imposed. We assumed the angle $\varphi$ to be constant, as a small rotation with a similar magnitude to $\delta \theta$ or $\delta \omega$ would not significantly alter the measured diffraction pattern [cf. Fig. 1(a)]. Similarly, the particle is assumed not to translate during the rockingcurve acquisition, which implies that the illumination conditions in the focused beam stay the same. This assumption is justified by considering that the typical displacement following a rotation would scale with the size of the rotation times the particle radius, and be of order $\delta \theta R<1 \mathrm{~nm}$.

The model is discretized as $W_{j i}$, where $j$ indexes the rocking-curve $(\theta)$ bins, and $i$ runs over the detector pixels. In a first step, the probability that the $k$ th experimental frame $K_{k i}$ corresponds to $\theta$ bin $j$ and to $\omega$ bin $l$ is calculated as a matrix $P_{j l k}$. Assuming Poisson counting statistics as the main noise contributor, these likelihoods can be expressed before normalization as $R_{j l k}$, which is conveniently calculated as its logarithm to avoid numerical instability. Taking the roll angle $\omega$ into account, the probability mass function of the Poisson distribution directly gives 


$$
\log R_{j l k}=\sum_{i}\left[\Omega_{l}\left(K_{k i}\right) \log \left(W_{j i}\right)-W_{j i}\right] .
$$

The $i$ index will be omitted in the following. In Eq. (1), $\Omega_{l}$ is a rotation operator which compensates for $\omega$ by shifting or rotating the image $K_{k}$ in the detector plane according to index $l$ before comparison with the model $W_{j}$. There is a fundamental difference between $\omega$ and $\theta$, in that the former can be approximately compensated within each two-dimensional diffraction pattern by redistributing intensities within the frame, whereas the latter cannot. The roll operator $\Omega_{l}$ can therefore be chosen as appropriate. Here we approximate the roll with a rotation along a ring in the detector plane, but for small roll angles a simple shift might suffice. For high count rates in $K_{k}$, iterative likelihood maximization tends to get stuck in local optima, which can be avoided using an annealing parameter $\beta$ that effectively smooths the likelihood landscape [25]. Since the probability that a measured diffraction pattern belongs somewhere in the three-dimensional model $W$ must equal one, the normalized probabilities are

$$
P_{j l k}=\frac{\left(R_{j l k}\right)^{\beta}}{\sum_{j, l}\left(R_{j l k}\right)^{\beta}} .
$$

In analogy with the original expand-maximize-compress algorithm [24], the resulting likelihood maximization update rule is

$$
W_{j}^{\prime}=\frac{\sum_{l, k} P_{j l k} \Omega_{l}\left(K_{k}\right)}{\sum_{l, k} P_{j l k}} .
$$

At the beginning of the assembly process, the probabilities of a frame belonging on either side of the rockingcurve center can become similar. This results in an artificial symmetry of the Bragg peak, and an X-shaped matrix $P_{j k}=\sum_{l} P_{j l k}$. In reality, the trajectory $\theta(k)$ is single valued, and the probability distribution must therefore be centered around some single angular bin $j$ for each frame $k$. To break this symmetry, a continuity bias can be imposed. Starting with the brightest frame at index $k_{\max }$, a Gaussian distribution of variance $n_{\sigma}^{2}$ elements along the $j$ index, centered on the most likely angular bin $j$ of its neighbor $\left(k-1\right.$ for $k>k_{\max }, k+1$ for $k<k_{\max }$ ), is then multiplied into $P_{j l k}$ before normalization.

In a final step the maximum extent of the particle in real space is used to constrain the volume model $W_{j}^{\prime}$. Specifically, the particle's autocorrelation function is obtained as the Fourier transform $\mathcal{F}$ of the diffraction volume. By confining the autocorrelation volume's extent along the direction corresponding to the rocking angle $\theta$, here denoted $x$, to $2 L$, the particle's maximum extent is restricted to $L$. The expression for the restricted autocorrelation function is then

$$
\mathcal{F}\left(W_{j}^{\prime \prime}\right)= \begin{cases}\mathcal{F}\left(W_{j}^{\prime}\right) & \text { where }|x| \leq L \\ 0 & \text { elsewhere }\end{cases}
$$

The inverse Fourier transform of Eq. (4) completes an iterative update $W_{j} \rightarrow W_{j}^{\prime \prime}$.

Results and validation.-Figure 2 demonstrates the described assembly algorithm with a numerical example. The truncated-octahedral particle illustrated in Fig. 1(a) was combined with an arbitrary phase, rendered from a spherical harmonic and a radial factor, and varying between -0.5 and $0.5 \mathrm{rad}$ [Fig. 2(a)]. Arbitrary $(\theta, \omega)$ trajectories around the reciprocal lattice point $G_{111}$ were generated [Fig. 2(c)] and diffraction patterns with applied Poisson noise generated [Fig. 2(b)]. The algorithm described above was then iteratively carried out for 100 iterations. Prior to

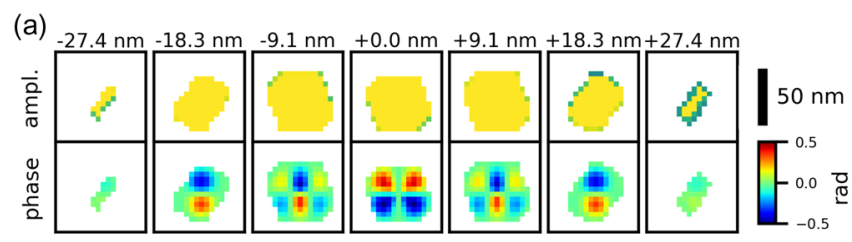

(b)

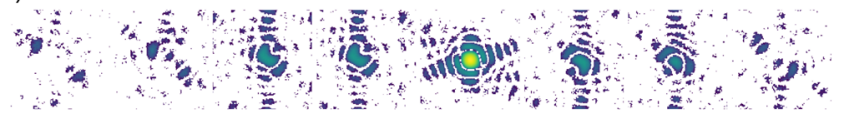

(c)

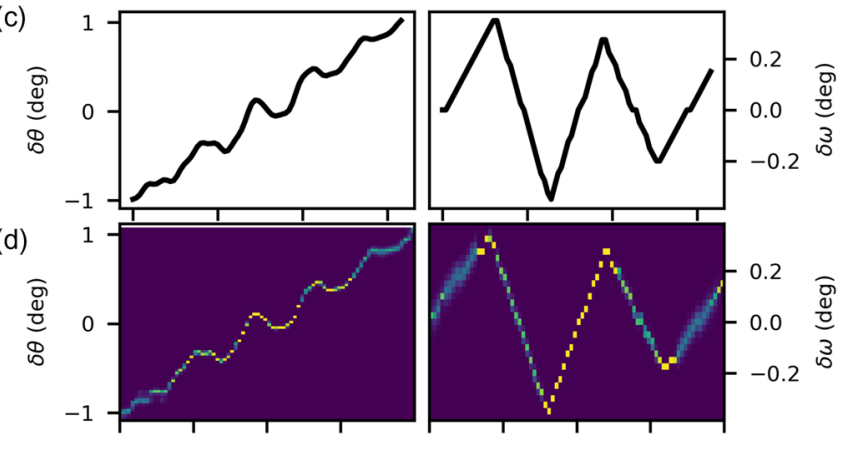

frame number $k$

(e)

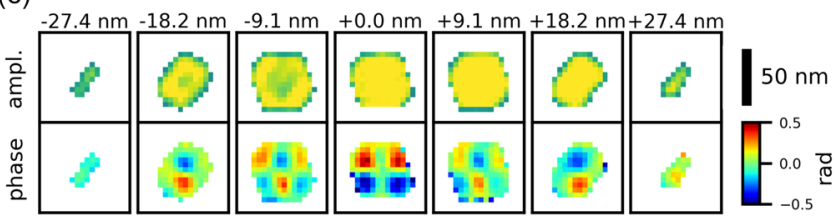

FIG. 2. A numerical experiment where data are simulated for arbitrary $(\theta, \omega)$ trajectories, assembled, and phase retrieved. (a) Input model particle [cf. Fig. 1(a)], described using slices of its phase and amplitude. (b) Selected frames illustrating the noise level, on an arbitrary and logarithmic scale. (c) The rocking and roll angles used as simulation input. (d) The matrices $P_{j k}=$ $\sum_{l} P_{j l k}$ (left) and $P_{l k}=\sum_{j} P_{j l k}$ (right) after 100 iterations of Eqs. (1)-(4). Both matrices are shown on a linear scale from 0 (navy) to 1 (yellow). (e) Reconstruction of the original particle by phase retrieval of the assembled diffraction volume, as in (a). 
assembly, the frames were prealigned along $\omega$ by applying the $\Omega_{l}$ operator such that their centers of mass lined up, saving computational effort by reducing the size of the $P_{j l k}$ matrix along $l$. Initial probabilities corresponding to a constant-velocity trajectory $\theta(k)$ and fixed $\omega(k)$ were used to generate the initial model according to Eq. (3). A soft bias with $6 \leq n_{\sigma} \leq 12$ was applied to $P_{j l k}$ as described above, in order to gently enforce a single-valued $\theta(k)$. A maximum particle dimension $D=60 \mathrm{~nm}$ constraint was applied, and the annealing parameter $\beta$ was initially set to $10^{-5}$ and then multiplied by $\sqrt{2}$ every 5 iterations. Figure 2(d) shows the angular probabilities from the assembly process, which recover the input trajectories very well (see Fig. S2 in Supplemental Material for residuals [26]).

The resulting diffraction volume model was phase retrieved with the PyNX software [27] where 1000 reconstructions were carried out, each with a constant shrinkwrap support threshold across the range 0.1-0.5 times the average sample amplitude [28]. Each reconstruction consisted of 600 relaxed averaged alternating reflection (RAAR) iterations [29] followed by 200 error-reduction cycles $[30,31]$. Before the support thresholding, the image was blurred using a Gaussian convolution kernel with $\sigma$ exponentially decreasing from 2 to 0.5 pixels from the first to the last RAAR step, following the PyNX default settings. The 20 reconstructions with the highest free log-likelihood metrics were selected and combined via mode decomposition, as described in Ref. [27]. The reconstructed particle faithfully reproduces the three-dimensional shape of the input, with the features of the truncated-octahedral envelope clearly seen. The reconstructed volume also shows the main features of the original phases. These numerical results are analyzed further in Supplemental Material, Figs. S3-S5 [26].
We now turn to the collection and analysis of experimental data. Shape-controlled Au nanoparticles of $60 \mathrm{~nm}$ diameter were synthesized, cleaned, and supported in a conductive carbon matrix as described elsewhere [32]. We have previously provided two-dimensional BCDI reconstructions of these model particles, and Ref. [15] contains transmission electron micrographs of the sample, from which an average distance between particles of $140 \mathrm{~nm}$ was found. Experimental BCDI data were collected by placing a deposit of $20 \mathrm{wt} \%$ particle-carbon mixture, tens of micrometers thick, on a $\mathrm{Si}_{3} \mathrm{~N}_{4}$ membrane, and positioning the sample in the focused $10 \mathrm{keV} \mathrm{x}$-ray beam. In this geometry, a few hundred particles are simultaneously illuminated, a small fraction of which might satisfy the Bragg condition at any time. The $\mathrm{x}$-ray focal spot ( $90 \mathrm{~nm}$ FWHM) is described in Ref. [20]. The detector used was a Merlin Quad (Quantum Detectors) of pixel size $55 \mu \mathrm{m}$, placed at $2 \theta_{111}$, corresponding to the reciprocal lattice point $\mathbf{G}_{111}$ for $\mathrm{Au}, 320 \mathrm{~mm}$ from the sample. The sample deposit was stepped through the beam, collecting bursts of 1000 images at $100 \mathrm{~Hz}(8 \mathrm{~ms}$ exposure, 2 ms readout) at each position. No sample rotation was used. In total, 2900 such bursts were collected and analyzed, of which several hundred contained visible diffraction patterns. The bursts containing the brightest transient diffraction spots were selected for further analysis. Since the particles are uniform in size [15], it can be assumed that the brightest rocking curves correspond to the most complete overlap of particle and beam.

Several uncontrolled rocking curves were analyzed by diffraction volume assembly and phase retrieval as described above for the simulated data, with only minor tuning of assembly parameters. Figure 3 shows data from one selected particle. In Fig. 3(a), the recovered rotational trajectories $\theta(k)$ and $\omega(k)$ are plotted, showing fairly (a)

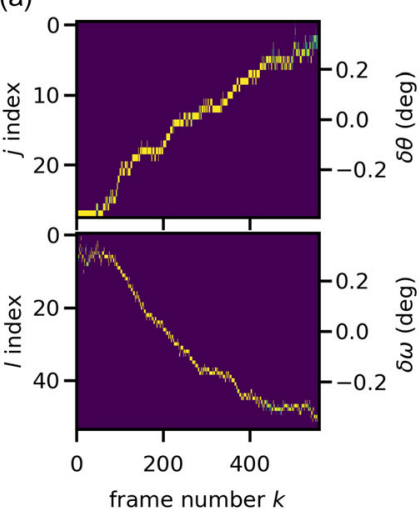

(b)

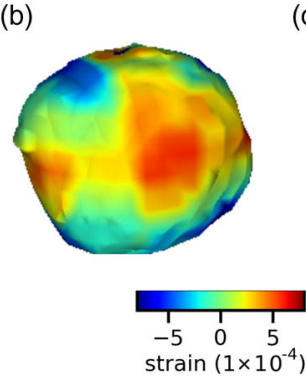

(d)

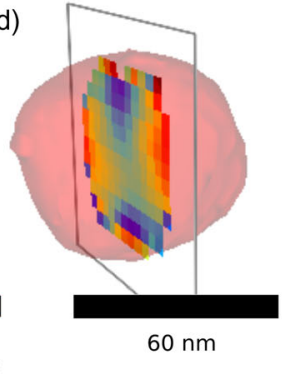

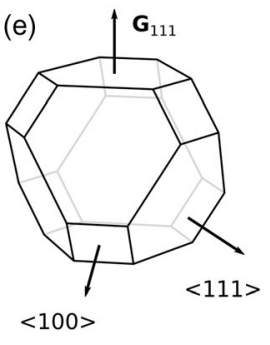

(e)

(c)

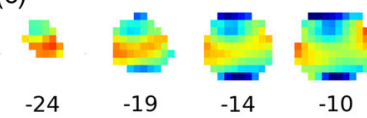

(f) Full-period resolution $\Delta r[\mathrm{~nm}]$
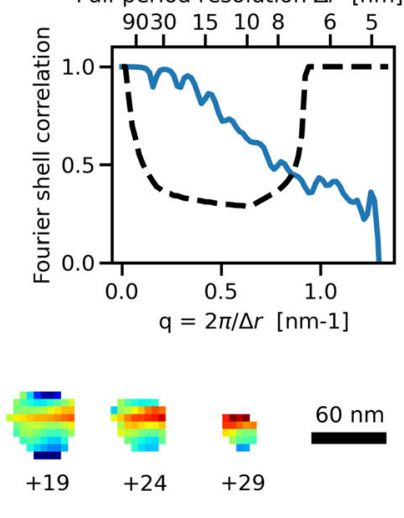

FIG. 3. Experimental results for a selected particle. (a) Assembly results presented as in Fig. 2(d). (b) Amplitude isosurface colored according to the (111) strain component on the surface. (c),(d) Cuts through the particle displaying the internal strain. (e) Orientation of the reconstructed particle as compared to the expected shape. (f) Fourier shell correlation analysis of the two half datasets, estimating the resolution of the final reconstruction. The dashed line shows the half-bit criterion [Eq. (17) of Ref. [33] ]. The criterion is corrected for the support by scaling the number of voxels in each shell by $(D / L)^{2}$, where $D$ and $L$ are the linear dimensions of support and phased volume, respectively [33]. 
smooth paths with fluctuating velocities. The reconstructed three-dimensional particle shape is shown in Fig. 3(b) and displayed as individual slices in Fig. 3(c). A cut perpendicular to the diffraction plane is indicated in Fig. 3(d). The reconstructed shape closely resembles the shape expected for these truncated-octahedral particles. By comparing the reconstructed sample to its ideal counterpart (Supplemental Material, Fig. S1 [26]) the $\varphi$ orientation can be determined, as shown for comparison in Fig. 3(e). The reconstructed voxel size is $(4.5 \mathrm{~nm})^{3}$, and a particle is around $12-15$ pixels across. At this pixel size and resolution, $\langle 111\rangle$ and sometimes $\langle 100\rangle$ facets can be discerned in the two-dimensional slices [Fig. 3(c) herein and Figs. S6-S14 in Supplemental Material [26] ], whereas a smooth three-dimensional model [Fig. 3(b)] merely reveals the general shape.

The colors in Fig. 3 reflect the lattice strain component along the Bragg vector $\mathbf{G}_{111}$ (in the figure's vertical direction). This strain component is obtained from the reconstructed phase $\phi$,

$$
\epsilon_{z z}=\frac{\partial u_{z}}{\partial z}=\frac{1}{\left|\mathbf{G}_{111}\right|} \frac{\partial \phi}{\partial z}
$$

where $z$ denotes the vertical direction [5]. The results show strain patterns that are strongest at the particle surfaces, and which decay toward its interior. A lattice compression along $z$ is seen at the top and bottom surfaces, while the particle perimeter shows a lattice expansion in the same direction. In terms of surface strain, this is consistent with a slight lateral surface lattice expansion. Such an expansion would give a positive $\epsilon_{z z}$ component around the particle perimeter and a negative contribution on the top and bottom as the lattice responds with a compressive elastic deformation normal to the surface.

Reconstructions of a number of independent particles show similar three-dimensional strain patterns, as shown in Supplemental Material, Figs. S6-S14 [26]. While this lends credence to the obtained results, individual particle reconstructions can also be validated by splitting one raw dataset in two, then assembling and phase retrieving each subset independently. This is routinely done in electron cryomicroscopy [33], and has also been used for XFEL singleparticle CDI [22]. We apply this validation method here, with the modification that frames $K_{k}$ are split into even and odd $k$, as randomizing the subsets can produce too large gaps in the angular trajectories. The reconstructed subsets can then be compared using Fourier shell correlation [34] to obtain an estimate of the actual resolution, as shown in Fig. 3(f) for the selected particle. The half-bit information threshold for the current detector geometry and assembly conditions (dashed line) indicates an average threedimensional resolution of around $8 \mathrm{~nm}[33,35]$, which is sufficient to reliably map the observed strain fields.
We have shown that it is possible to assemble a coherent diffraction volume from rocking curves with unknown angular trajectories. These volumes can be phase retrieved, producing verifiable three-dimensional shape and strain maps of crystalline nanoparticles. In general, this ability allows applying BCDI to new conditions not limited to beam-induced motions, including, for example, dynamic samples or particles in suspension, as long as the frame rate is fast compared to the rotation. For beam-induced rotations in particular, the method presented can significantly increase BCDI's robustness. In relaxing the tolerances on the rocking angle, the range of applicability of BCDI is extended to smaller and more technologically relevant particles which are prone to beam-induced sample rotation. By overcoming the rotation stability problems in high-flux experiments we therefore hope that this Letter heralds the use of fourth generation synchrotrons for BCDI to their full potential.

All code and raw data described in this Letter are deposited and available through Ref. [36].

Research conducted at MAX IV, a Swedish national user facility, is supported by the Swedish Research council under Contract No. 2018-07152, the Swedish Governmental Agency for Innovation Systems under Contract No. 2018-04969, and Formas under Contract No. 2019-02496. This work has also received funding from the ÅForsk Foundation (Contract No. 17-408), the European Research Council (ERC) under the European Union's Horizon 2020 research and innovation programme (Contract No. 801847), from the Olle Engkvist Foundation, from the Swedish Research council (Contract No. 201800234), and from NanoLund.

*alexander.bjorling@maxiv.lu.se

[1] M. A. Pfeifer, G. J. Williams, I. A. Vartanyants, R. Harder, and I. K. Robinson, Three-dimensional mapping of a deformation field inside a nanocrystal, Nature (London) 442, 63 (2006).

[2] I. Robinson and R. Harder, Coherent x-ray diffraction imaging of strain at the nanoscale, Nat. Mater. 8, 291 (2009).

[3] F. Berenguer, P. Godard, M. Allain, J.-M. Belloir, A. Talneau, S. Ravy, and V. Chamard, X-ray lensless microscopy from undersampled diffraction intensities, Phys. Rev. B 88, 144101 (2013).

[4] V. Favre-Nicolin, F. Mastropietro, J. Eymery, D. Camacho, Y. M. Niquet, B. M. Borg, M. E. Messing, L.-E. Wernersson, R. E. Algra, E. P. A. M. Bakkers, T. H. Metzger, R. Harder, and I. K. Robinson, Analysis of strain and stacking faults in single nanowires using Bragg coherent diffraction imaging, New J. Phys. 12, 035013 (2010).

[5] M. C. Newton, S. J. Leake, R. Harder, and I. K. Robinson, Three-dimensional imaging of strain in a single $\mathrm{ZnO}$ nanorod, Nat. Mater. 9, 120 (2010). 
[6] A. Ulvestad, A. Singer, H.-M. Cho, J. N. Clark, R. Harder, J. Maser, Y.S. Meng, and O. G. Shpyrko, Single particle nanomechanics in operando batteries via lensless strain mapping, Nano Lett. 14, 5123 (2014).

[7] A. Ulvestad, A. Singer, J. N. Clark, H. M. Cho, J. W. Kim, R. Harder, J. Maser, Y.S. Meng, and O. G. Shpyrko, Topological defect dynamics in operando battery nanoparticles, Science 348, 1344 (2015).

[8] A. Ulvestad, K. Sasikumar, J. W. Kim, R. Harder, E. Maxey, J. N. Clark, B. Narayanan, S. A. Deshmukh, N. Ferrier, P. Mulvaney, S. K. R. S. Sankaranarayanan, and O. G. Shpyrko, In situ 3D imaging of catalysis induced strain in gold nanoparticles, J. Phys. Chem. Lett. 7, 3008 (2016).

[9] A. Ulvestad and A. Yau, The self-healing of defects induced by the hydriding phase transformation in palladium nanoparticles, Nat. Commun. 8, 1376 (2017).

[10] Y. Liu, P. P. Lopes, W. Cha, R. Harder, J. Maser, E. Maxey, M. J. Highland, N. M. Markovic, S. O. Hruszkewycz, G. B. Stephenson, H. You, and A. Ulvestad, Stability limits and defect dynamics in Ag nanoparticles probed by Bragg coherent diffractive imaging, Nano Lett. 17, 1595 (2017).

[11] F. Meirer and B. M. Weckhuysen, Spatial and temporal exploration of heterogeneous catalysts with synchrotron radiation, Nat. Rev. Mater. 3, 324 (2018).

[12] D. Kim, M. Chung, J. Carnis, S. Kim, K. Yun, J. Kang, W. Cha, M. J. Cherukara, E. Maxey, R. Harder, K. Sasikumar, S. K. R. S. Sankaranarayanan, A. Zozulya, M. Sprung, D. Riu, and H. Kim, Active site localization of methane oxidation on Pt nanocrystals, Nat. Commun. 9, 3422 (2018).

[13] D. Kim, M. Chung, S. Kim, K. Yun, W. Cha, R. Harder, and H. Kim, Defect dynamics at a single Pt nanoparticle during catalytic oxidation, Nano Lett. 19, 5044 (2019).

[14] M. Abuin, Y. Y. Kim, H. Runge, S. Kulkarni, S. Maier, D. Dzhigaev, S. Lazarev, L. Gelisio, C. Seitz, M. I. Richard, T. Zhou, V. Vonk, T. F. Keller, I. A. Vartanyants, and A. Stierle, Coherent X-ray imaging of CO-adsorption-induced structural changes in Pt nanoparticles: Implications for catalysis, ACS Appl. Nano Mater. 2, 4818 (2019).

[15] A. Björling, D. Carbone, F. J. Sarabia, S. Hammarberg, J. M. Feliu, and J. Solla-Gullón, Coherent Bragg imaging of $60 \mathrm{~nm}$ Au nanoparticles under electrochemical control at the NanoMAX beamline, J. Synchrotron Radiat. 26, 1830 (2019).

[16] G. J. Williams, M. A. Pfeifer, I. A. Vartanyants, and I. K. Robinson, Three-Dimensional Imaging of Microstructure in Au Nanocrystals, Phys. Rev. Lett. 90, 175501 (2003).

[17] J. W. Kim, A. Ulvestad, S. Manna, R. Harder, E. Fohtung, A. Singer, L. Boucheron, E. E. Fullerton, and O. G. Shpyrko, Observation of x-ray radiation pressure effects on nanocrystals, J. Appl. Phys. 120, 163102 (2016).

[18] M. Liang, R. Harder, and I. Robinson, Radiation-driven rotational motion of nanoparticles, J. Synchrotron Radiat. 25, 757 (2018).

[19] I. Calvo-Almazán, M. Allain, S. Maddali, V. Chamard, and S. O. Hruszkewycz, Impact and mitigation of angular uncertainties in Bragg coherent x-ray diffraction imaging, Sci. Rep. 9, 6386 (2019).
[20] A. Björling, S. Kalbfleisch, M. Kahnt, S. Sala, K. Parfeniukas, U. Vogt, D. Carbone, and U. Johansson, Ptychographic characterization of a coherent nanofocused X-ray beam, Opt. Express 28, 5069 (2020).

[21] N. D. Loh, M. J. Bogan, V. Elser, A. Barty, S. Boutet, S. Bajt, J. Hajdu, T. Ekeberg, F. R. N. C. Maia, J. Schulz, M. M. Seibert, B. Iwan, N. Timneanu, S. Marchesini, I. Schlichting, R. L. Shoeman, L. Lomb, M. Frank, M. Liang, and H. N. Chapman, Cryptotomography: Reconstructing 3D Fourier Intensities from Randomly Oriented SingleShot Diffraction Patterns, Phys. Rev. Lett. 104, 225501 (2010).

[22] T. Ekeberg et al., Three-Dimensional Reconstruction of the Giant Mimivirus Particle with an X-Ray Free-Electron Laser, Phys. Rev. Lett. 114, 098102 (2015).

[23] H. N. Chapman et al., Femtosecond diffractive imaging with a soft-x-ray free-electron laser, Nat. Phys. 2, 839 (2006).

[24] N. T. D. Loh and V. Elser, Reconstruction algorithm for single-particle diffraction imaging experiments, Phys. Rev. E 80, 026705 (2009).

[25] K. Ayyer, T. Y. Lan, V. Elser, and N. D. Loh, Dragonfly: An implementation of the expand-maximize-compress algorithm for single-particle imaging, J. Appl. Crystallogr. 49, 1320 (2016).

[26] See Supplemental Material at http://link.aps.org/ supplemental/10.1103/PhysRevLett.125.246101 for figures supporting the method validation, and additional particle reconstructions

[27] V. Favre-Nicolin, S. Leake, and Y. Chushkin, Free loglikelihood as an unbiased metric for coherent diffraction imaging, Sci. Rep. 10, 2664 (2020).

[28] S. Marchesini, H. He, H. N. Chapman, S. P. Hau-Riege, A. Noy, M. R. Howells, U. Weierstall, and J. C. H. Spence, $\mathrm{X}$-ray image reconstruction from a diffraction pattern alone, Phys. Rev. B 68, 140101(R) (2003).

[29] D. R. Luke, Relaxed averaged alternating reflections for diffraction imaging, Inverse Probl. 21, 37 (2005).

[30] R. W. Gerchberg and W. O. Saxton, A practical algorithm for the determination of the phase from image and diffraction plane pictures, Optik (Stuttgart) 35, 237 (1972).

[31] J. R. Fienup, Phase retrieval algorithms: A comparison, Appl. Opt. 21, 2758 (1982).

[32] H. Erikson, A. Sarapuu, K. Tammeveski, J. Solla-Gullón, and J. M. Feliu, Shape-dependent electrocatalysis: Oxygen reduction on carbon-supported gold nanoparticles, ChemElectroChem 1, 1338 (2014).

[33] M. Van Heel and M. Schatz, Fourier shell correlation threshold criteria, J. Struct. Biol. 151, 250 (2005).

[34] G. Harauz and M. Van Heel, Exact filters for general geometry three dimensional reconstruction, Optik (Stuttgart) 73, 146 (1986).

[35] K. Ayyer, A. J. Morgan, A. Aquila, H. DeMirci, B. G. Hogue, R. A. Kirian, P. L. Xavier, C. H. Yoon, H. N. Chapman, and A. Barty, Low-signal limit of x-ray single particle diffractive imaging, Opt. Express 27, 37816 (2019).

[36] A. Björling, https://cxidb.org/id-151.html. 